\title{
Effect Neural Networks on Selected Feature by Meta Heuristic Algorithms
}

\author{
Maysam Toghraee ${ }^{\text {a }}$, Farhad rad ${ }^{\mathrm{b}}$, Hamid parvin ${ }^{\mathrm{a}, \mathrm{b}}$ \\ ${ }^{a}$ Faculty of engineering, department of computer science, Islamic Azad University, Kohgilouye-va- \\ Boyerahmad, Isfahan and 8661854381, Iran \\ ${ }^{b}$ Faculty of engineering, department of computer science, Islamic Azad University, Kohgilouye-va- \\ Boyerahmad, Yasouj, Iran \\ ${ }^{a, b}$ Faculty of engineering, department of computer science, Islamic Azad University, Kohgilouye-va- \\ Boyerahmad, Yasouj, Iran
}

\begin{abstract}
Feature selection is one of the issues that have been raised in the discussion of machine learning and statistical identification model. We have provided definitions for feature selection and definitions needed to understand this issue, we check. Then, different methods for this problem were based on the type of product, as well as how to evaluate candidate subsets of features, we classify the following categories. As in previous studies may not have understood that different methods of assessment data into consideration, We propose a new approach for assessing similarity of data to understand the relationship between diversity and stability of the data is selected. After review and meta-heuristic algorithms to implement the algorithm found that the cluster algorithm has better performance compared with other algorithms for feature selection sustained.
\end{abstract}

Index Terms: Feature selection, data mining, algorithm cluster, heuristic methods.

(C) 2016 Published by MECS Publisher. Selection and/or peer review under responsibility of the Research Association of Modern Education and Computer Science

\section{Introduction}

Manual processing of data is impracticable because of Fast growing technologies. Even worse, machine learning techniques and knowledge extraction seem to be unable against this big data base. Vast dimensions management is one of the common challenges for extracting knowledge and machine learning. Feature selection methods is one of the most challenging and the most important activities in developing machine learning and patterns recognition. Feature selection is one of the issues which has been discussed in machine learning and also pattern statistical recognition. This issue is important in many usages(e.g. classification),

* Corresponding author. Tel.: 09304586038

E-mail address: may.toghraee@yahoo.com 
since there are a lot of features in these usages. Many of them are useless or are not informative. Eliminating these features does not change the informative content but it affects on calculating feature of the mentioned usage Also it helps to save much useless information with useful data. Not elimination of the waste features makes some dimensional problems. Dimensional problem says that when the dimensions increase, it is possible that the 2 data (or sample) get far from each other. It means that the higher the dimensions(features), probably the most distance between them randomly. As the result, those samples are affected by the dimension and the distance between those samples are estimated much more. It makes the distance between both samples less representing the real distances. So, the quality of classifying or clustering are unpleasantly unreal and drop. It can be stated in another way. It can be said that some clusters or branches in feature's atmosphere are more coherent with some special features; Three general ways have been submit to overcome the above dimension problem: (a) using subspaces determined for clusters or branches by user, (b) using feature selection methods or decreasing dimensions like analyzing main factors and finally (c) using subspace clustering or subspace classifying methods. We discuss about the feature selection methods (b) in this report. A lot of solutions and algorithm have been represented for feature selection issue. A lot of solutions and algorithms have been represented for feature selection issue, some of which are 30 or 40 years old. The problem about algorithms when represented was their calculating feature. However, fast computers and big saving sources have made this problem unimportant, beside, big data sets for new issues has made it important to find a fast algorithm for this issue. Feature selection has 2 types:

a) Supervised feature: Labels are used during feature selection algorithm(Zhao \&Liu 2007)

b) Unsupervised feature :Labels are not used during feature selection algorithm(G.D 2008)

Research domain is just limited to the supervised feature selection while labels are used during feature selection algorithm.

Purpose of feature selection is decreasing the dimensions of a set to a smaller subset of the set's features. This subset of main features is known as the best subset of features; A target function is used as being better factor which can be different targets according to the primary hypothesis. This being better can be measured, for example accuracy in classifying. We are seeking for the below targets using local searching methods and imitating the nature searching algorithm:

a) Discuss about the feature selection efficiency methods.

b) Explain the assessing algorithm of this research

c) Looking for tests and results from these methods using real data sets.

\section{Efficiency Function Methods}

Different types of efficiency function for various subsets includes (1) wrapper methods, (2) embedded methods, (3) filter methods.

Wrapper methods: To asses each of the candidate feature subsets, a classifying model is done on those features(instruct and produce). We consider the accuracy of the trained classifier on a distinct experimental set as the efficiency amount of that candidate subset. It is clear that however these methods are suitable for efficiency, they are slow and are complicated in calculation. Since these methods teach a classifying model for each assessment of efficiency function, these methods are appropriate for the environments with subset generator similar to the optimization algorithm of nature imitation.

Utilized methods of classified model during learning the educational data set, feature selection is done implicitly. An example of this kind of feature selection methods is decision tree classifying model. As these methods are explicitly part of feature selection methods, we do not use these methods.

Filter methods, Set of different subsets of features are placed according to a factor. 
Then we can choose their best. The problem is that these methods try to find the best subset of features out of candidate subset $2^{n}$. Producing all of them is hard and inapplicable but since calculating the efficiency function in these methods is mostly fast(the accuracy of classified model is not on selected features), they are appropriate for environments with subset generators similar to optimization algorithms of nature imitation .

\subsection{Efficiency Function}

To calculate the efficiency function, we should first calculate the relationship of each feature with other features and label.

After calculation of each feature with other features and label, selecting features is done regarding to relationship amount of each feature with other features and labels. To do this, methods depended on evolutional algorithms have been represented for selecting subsets of features, in this chapter we discuss about the efficiency function of this algorithms. We are seeking for 2 targets in feature selecting. First, an evolutional algorithm is looking for subset of features which has the most relationship with label and second the selecting features have the least relationship with other selecting features. Both are demonstrated in evolutional algorithms of efficiency function explicitly.

Now, we use the following relation which shows the variation of selecting features and label similarity of selecting features to calculate the efficiency of this chromosome.

$$
f i t_{c h}=\sum_{i=1}^{f} \operatorname{lesser}\left(\max _{k=1}^{f}\left(\left|\operatorname{cor}\left(X_{i}, X_{k}\right)\right| \times \operatorname{and}\left(\operatorname{ch}_{i}, c h_{k}\right)\right), t h_{2}\right) \times \alpha+\operatorname{greater}\left(\left|\operatorname{cor}\left(X_{i}, T\right)\right|, t h_{1}\right)
$$

Where $\mathrm{f}$ is the amount of features and $\alpha$ is the big positive number, $\mathrm{th}_{1}$ and $\mathrm{th}_{2}$ are two thresholds which should be adjusted by the user, $\mathrm{ch}_{\mathrm{j}}$ shows Ith of chromosome, ${ }_{\text {|I }}$ shows the absolute value, $\operatorname{cor}\left(\mathrm{X}_{\mathrm{j}}, \mathrm{X}_{\mathrm{k}}\right)$ shows the relationship of $\mathrm{I}$ th and $\mathrm{k}$ th features, and $\left(\mathrm{ch}_{\mathrm{j}}, \mathrm{ch}_{\mathrm{k}}\right)$ show the logical operator (output is 1 when both inputs are 1 ,otherwise function output is 0$),(a, b)$ is greater than 1 if $a \geq b$,otherwise function output is 0 and function $(a, b)$ is less than 1 , if $a \leq b$. otherwise function output is 0 .

\section{Evaluation Algorithms}

\subsection{Genetics Algorithm}

The main idea of evolutional algorithms were presented in 1960 by Ritchenberg. Genetic algorithms are derived from this type of algorithm. In fact the computer search methods based on optimization algorithms based on the genetic structure of chromosome's by john Holland(1970) was introduced at the university of Michigan(A.mehdi;1386). The most extensive definitions of genetic algorithm are from goldberg's book:" Genetic algorithms is machine learning model, its behavior is an example of the evolutionary processes in nature". Genetic algorithm is one the strongest methods derived from the nature which seeks for the problem randomly. This search is seeking for creating better answers in each generation compared to the answers of previous generations which represents one of the best forms of number optimization on the issues of science and engineering (masoudian \& et al;2007). We explain the genetics algorithm steps based on (C . Meyers \&et al; 1996): First we answer the question by defining a chromosome structure (coding). Introducing the fitness function, we explain the quality of the given answers in each chromosome numerically. Then we generate some chromosomes randomly (or semi-random. And the chromosomes are known as the initial population. We have some answers for the problem in this step which have lower quality. The quality of each chromosome of the population is specified according to fitness function we specified. Now we select two chromosomes for reproduction using an appropriate method (A method in which the probability of chromosome selection with better fitness amount is more than a chromosome with less fitness amount). Then using these two chromosome, we create a new chromosome (mating). We change some genes of some chromosomes having a specified probability. Selecting, recombination and mutation steps make a new population of chromosomes (new 
generation). If the chromosomes tend to the demanded answer, reproduction process stops. Otherwise creating a generation out of the previous generation continues until we reach to a desired answer or ending the algorithm, condition.

\subsection{Hill Climbing Algorithm}

In Hill climbing algorithm, at first an answer to the question is generated randomly. Then in a loop until the stop condition is not established for the algorithms, a number of neighbors are generated for the current mode of production and the best one is chosen among them and replaces the current mode. (of course, another definition for the hill climbing has been stated). In general, the optimality of the answer to the algorithm is local. To run Hill climbing algorithm we need two functions: performance function and neighbor function. The performance function determines optimality of the answer. 8 minister of guards on board pairs queens returns. Neighbor function also produces current mode neighbors. In problem 8 queens are for generating neighbor modes, each of them are chosen and move once upward or downward. This means that in the worst mode of each case, there will be 16 neighboring modes that in each repetition of the loop as the best answer will be replaced neighbors. ends when there is not a better mode compared to the current mode.

\subsection{Clustering algorithm}

A general trend for clustering process includes the following steps:

1. Displaying the patterns that usually involves selecting or extracting the feature.

2. Defining an assessment criteria of the similarity according to the data domain.

3. Clustering or Grouping process.

4. Summarization of data if needed.

5. Validation of system.

Displaying the patterns refers to the number of classes, available samples and the number, type and scale of features in a clustering algorithm. Some of these data are not controlled by the user. Selecting feature, Identifying process of a subset is one of the most effective features for using in clustering and feature extracting is, the process of changing some available features and generating new features. Both of these techniques are used in order to achieve a suitable set of features and enhancing the performance of clustering. Adjacent of samples usually is measured by a function of distance between the pair of input patterns. Various criterion are used to measure distances in various fields. A simple measuring criteria such as Euclidean distance is usually used to represent the dissimilarity between the two models. Identifying conceptual similarities between the patterns, evaluation criteria could no longer be used. Grouping step has various methods. The results of the clustering can be in from of hard segmentation (dividing data within separate groups) or phase segmentation (each sample is located in various groups with different membership grades). Hierarchical clustering algorithm represent nested chains of different divisions on the basis of dividing and combining clusters by similarity function. Non-hierarchical clustering algorithm gives a division of the samples. There are other techniques, such as possibility clustering and graph theory clustering. Summarization of data is the extraction process of a precise and simple of the original data set. In clustering, a sample of data summarization could be a short description for each cluster is usually referred to as per-pattern model. For example, the center of gravity for each cluster could be a brief description of the sample within that cluster.

\section{Evaluation Methods}

In this section, the results of applying the proposed method on different data sets and used parameters has been reported. In this section, analysis and interpretation of the results and efficiency of the proposed algorithm 
also briefly discussed. In this research validation includes experimental validation. In experimental validation, the efficiency of proposed algorithm is discussed in comparison with other algorithm in several real data sets. UCI standard data sets are the used datasets (it is on the machine learning website) is that almost all the result of recent studies in the field of data mining in the world are reported using this data set. These actual data are often standard. Experimental results of the proposed method and other methods with the world's valid criterion such as error size the feature set size and chosen will be reported.

Table.1 Datasets used in the First Experiment In This Thesis. Starred Data set"*" are Real Data Sets.

\begin{tabular}{|c|c|c|c|c|}
\hline Dataset Name & \# of data items & \# of features & \# of classes & Data distribution per clusters \\
\hline Breast Cancer* & 404 & 9 & 2 & $444-239$ \\
\hline Bupa* $^{*}$ & 345 & 6 & 2 & $145-200$ \\
\hline Glass* $^{*}$ & 214 & 9 & 6 & $70-76-17-13-9-29$ \\
\hline Galaxy* & 323 & 4 & 7 & $51-28-46-38-80-45-35$ \\
\hline SAHeart* & 462 & 9 & 2 & $160-302$ \\
\hline Ionosphere* & 351 & 34 & 2 & $126-225$ \\
\hline Iris* & 150 & 4 & 3 & $50-50-50$ \\
\hline Wine* & 178 & 13 & 3 & $59-71-48$ \\
\hline Yeast* & 1484 & 8 & 10 & $463-5-35-44-51-163-244-429-20-30$ \\
\hline
\end{tabular}

It should be mentioned that all results represented in this thesis are achieved, because the test results are so strong and extendable, with an average of 10 separate performance. In the first experiments have been performance on several real data sets. The actual data sets have been derived from machine learning website (Newman, 1998). These data sets is presented in the table above. properties of this dataset with zero mean and variance one, $\mathrm{N}(0,1)$ are standard. Thresholds $t h_{1}$ and $t h_{2}$ changing from 0,1 to 0,9 and training set size from 10 percent to 90 percent, different levels of accuracy are achieved. Notice that each performance is done 10 times repeatedly. In all cases the maximum population size should be adjusted on 50 and the maximum generation size on 50 .

\section{Results from Algorithms}
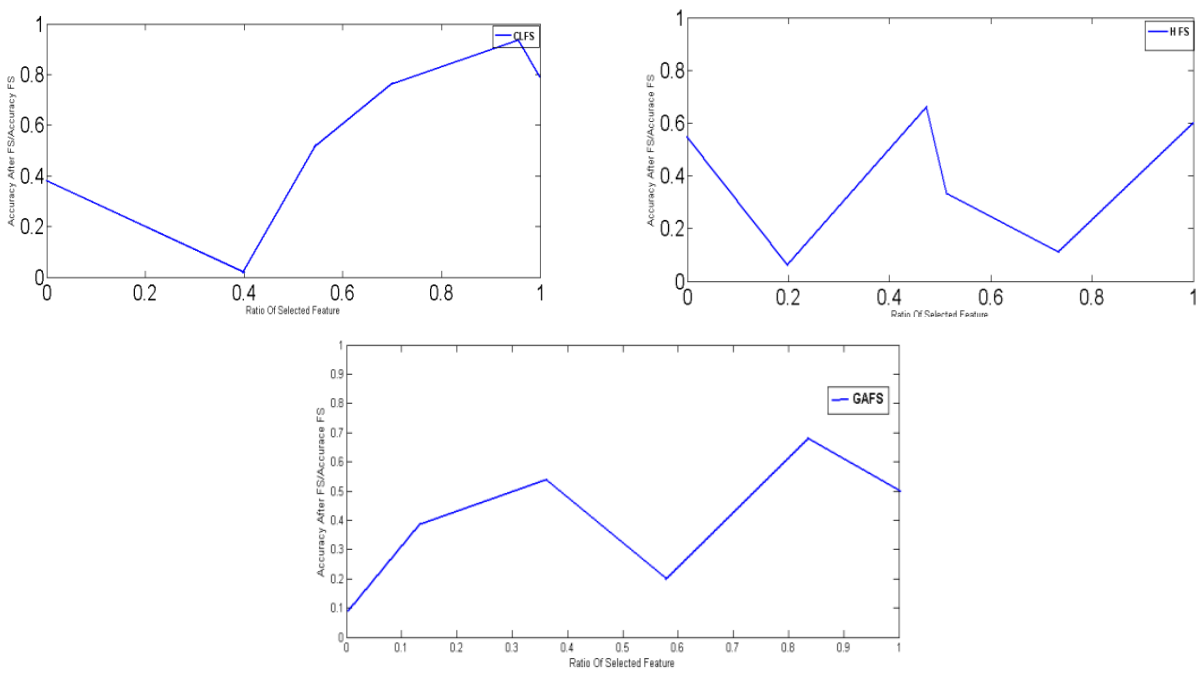

Fig.1. Average Results Carefully Selected Revelation of Various Features on Different Datasets with Different Percentages of Optional Features 
AS shown, the accuracy of cluster method is higher than other methods and Genetic algorithm is in second rank.
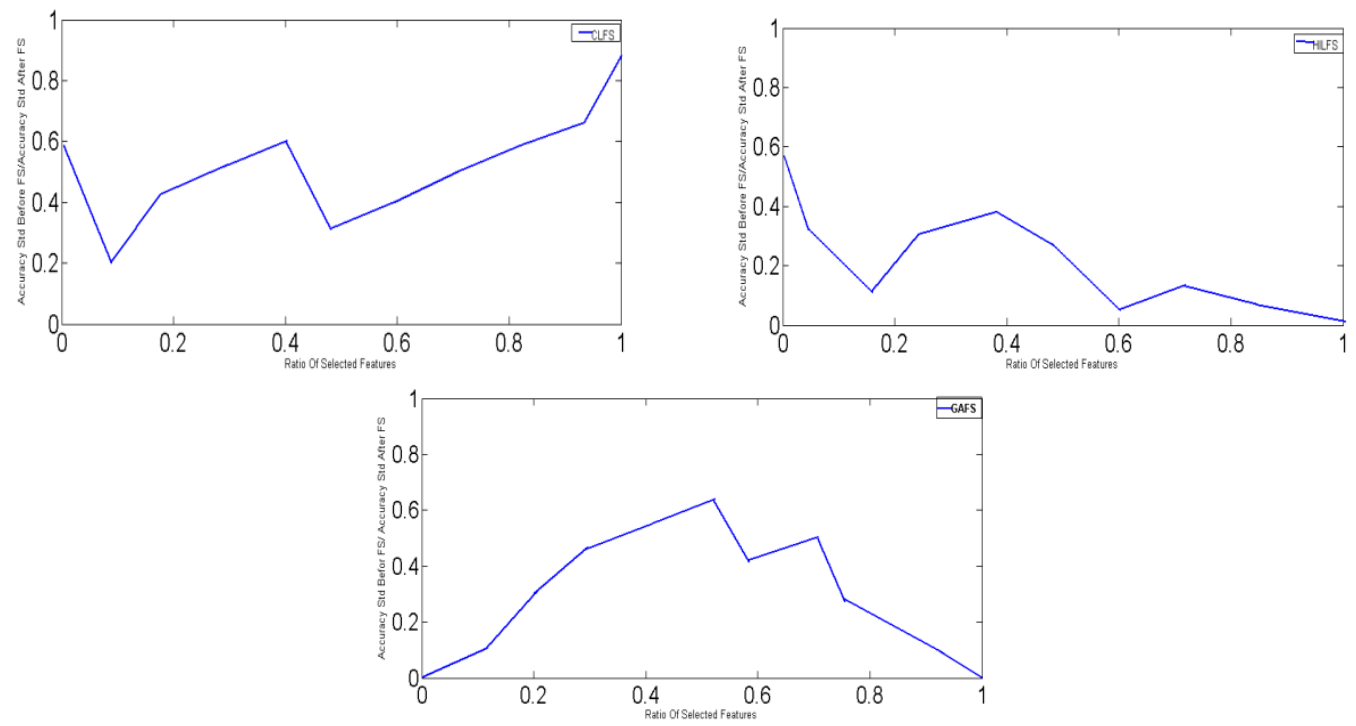

Fig.2. Average Standard Deviation of the Accuracy of the Results of Heuristic Methods to Select Different Features on Different Datasets with Different Percentages of Optional Features.

The standard deviation of accuracy of cluster method is acceptable compared to other methods and Genetic algorithm is in second rank.
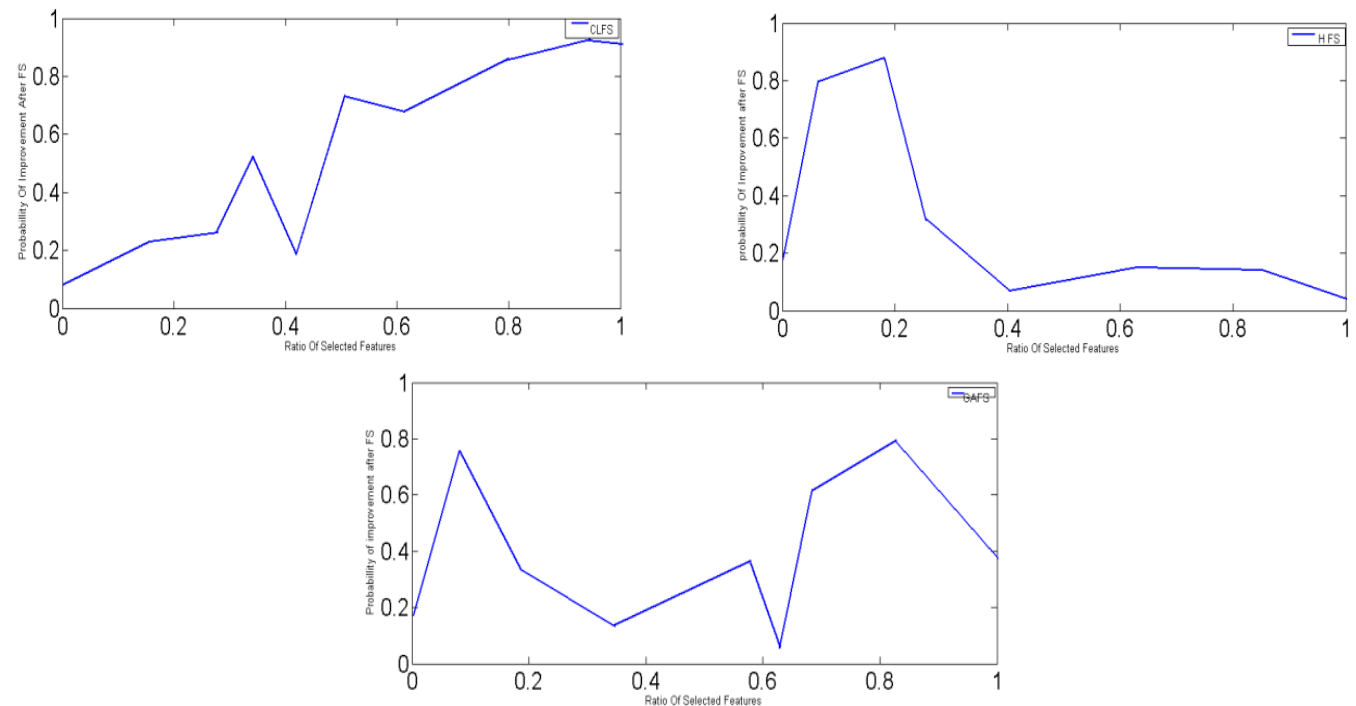

Fig.3. Likely to Improve the Accuracy Rate of a Consensus of Neural Networks after the Feature Selection Method. 
As shown, feature selection of cluster algorithm by selection of 80 to $100 \%$ of features maximizes the improvement probability in learning rate. And feature selection of Genetic method by selection of 70 to $80 \%$ of features maximizes improvement in learning rate.

\section{Conclusions}

In this study, the efficiency of the proposed algorithm, advantages, challenges and innovations have been discussed briefly. A set of feature selecting method based on the collective intelligence methods has been presented. It was shown experimentally that these methods can have no decrease in classifying quality, selecting almost $80 \%$ of features. Beside, these experiments showed that clustering method is the best way in finding appropriate features for classifying.

\section{Reference}

[1] Dy J. Unsupervised feature selection. Computational Methods of Feature Selection, (2008). pages 19-39.

[2] Guyon I and Elise A. An introduction to variable and feature selection. Journal of Machine Learning Research, (2003).3:1157-1182.

[3] Jain k., Dubes R. C. Algorithms for Clustering Data, Prentice Hall, Englewood Cliffs. (1988).

[4] Lampinen $\mathbf{J}$ and Laksone $\mathbf{J}$ and Oja E. Pattern recognition. In editor, Image Processing and Pattern Recognition, volume 5 of Neural Network Systems Techniques and Applications, (1998). pages 1- 59. Academic Press.

[5] Ladha L and Scholar R, Depa T, LFeature Selection Methods and Algorithms, International Journal on Computer Science and Engineering (IJCSE), Vol. 3, No. 5, (2011). pp. 1787-1797.

[6] Marki F and Vogel M and Fischer M. "Process Plan optimization using a Genetic Algorithm", PATAT, (2006), pp. 528-531. ISBN 80-210-3726-1.

[7] Marki F and Vogel M and Fischer M. "Process Plan optimization using a Genetic Algorithm", PATAT, (2006). pp. 528-531. ISBN 80-210-3726-1.

[8] Masoudian S and ESTEKI A., "Design schedule automatically using genetic algorithm ", thesis, university of Isfahan. (1386).

[9] Matlab version 7.4.0.287(R2012a), 29 january 2012, U.S.Patents Carol Meyers and James B. Orlin, (2011), "Very Large-Scale Neighborhood Search Techniques in Timetabling Problems", PATAT 2011, pp. 36-52. ISBN 80-210-3726-1.

[10] Mehdi A." Introduction to genetic algorithm and application", Tehran: Bell Press naghos. (1386).

[11] Murata S and Kurova H. Self-Organization of Biological Systems. (2012).

[12] Neumann J, C and Schnar G. S. Combined SVM-based feature selection and classification, Machine Learning, (2005). Vol. 61, No. 3, pp. 129 - 150.

[13] Perzina R. "Solving the University Timetabling Problem with Optimized Enrolment of Students by aParallel Self-adaptive Genetic Algorithm", (2006). PATAT 2006, pp. 264-280. ISBN 80-210-3726-1.

[14] Susana M and Vieira J and Sousa M.C. Fuzzy criteria for feature selection, Fuzzy Sets and Systems, (2012).Vol. 189, No. 1, pp. 1-18.

[15] Zhao Z and Liu H. Semi-supervised feature selection via spectral analysis. In Proceedings of SIAM International Conference on Data Mining (SDM). (2007). 


\section{Authors' Profiles}

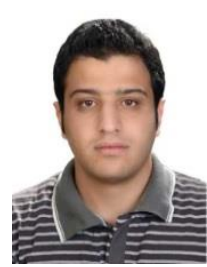

Maysam Toghraee first author is this article. Maysam toghraee in a family toghraeee semiromi was born in 1987 in semirom and primary and secondy education the in city end and after goes in Esfahan in university shahid ahrafi esfahani succeeded to a bachelor's Degree in since computer in 2010. and went in university yasouj in field of engineering software computer continuing education, and my own thesis in the field of data mining with matlab software was defencs.

H. parvin advisor and second author phd artificial intelligence of iran university of science technology and his expertise is data mining.

F. Rad supevisor and third author phd architecture computer science\& research technology.

How to cite this paper: Maysam Toghraee, Farhad rad, Hamid parvin,"Effect Neural Networks on Selected Feature by Meta Heuristic Algorithms", International Journal of Mathematical Sciences and Computing(IJMSC), Vol.2, No.3, pp.41-48, 2016.DOI: 10.5815/ijmsc.2016.03.04 